\title{
Re-organization of graphite surfaces into carbon micro- and nanoparticles under high flux hydrogen plasma bombardment
}

\author{
Kirill Bystrov, ${ }^{1, a)}$ Lenze van der Vegt, ${ }^{1}$ Gregory De Temmerman, ${ }^{1}$ Cécile Arnas, ${ }^{2}$ and \\ Laurent Marot $^{3}$ \\ 1) FOM Institute DIFFER - Dutch Institute for Fundamental Energy Research, \\ Association EURATOM-FOM, Partner in the Trilateral Euregio Cluster, \\ P.O. Box 1207, NL-3430 BE Nieuwegein, The Netherlands \\ ${ }^{2)}$ Laboratoire de Physique des Interactions Ioniques et Moléculaires (PIIM), \\ CNRS/Aix-Marseille Université, F-13397 Marseille, France \\ 3) Department of Physics, University of Basel, Klingelbergstrasse 82, CH-4056 Basel, \\ Switzerland
}

(Dated: 5 October 2012)

Fine-grain graphite samples were exposed to high density low temperature $\left(n_{e} \sim\right.$ $10^{20} \mathrm{~m}^{-3}, T_{e} \sim 1 \mathrm{eV}$ ) hydrogen plasmas in the Pilot-PSI linear plasma generator. Re-deposition of eroded carbon is so strong that no external precursor gas injection is necessary for deposits to form on the exposed surface during the bombardment. In fact, up to $90 \%$ of carbon is re-deposited, most noticeably in the region of highest particle flux. The re-deposits appear in the form of carbon microparticles of various sizes and structures. Discharge parameters influence the efficiency of the re-deposition processes and the particle growth rate. Under favourable conditions the growth rate reaches $0.15 \mu \mathrm{m} / \mathrm{s}$. We used high resolution scanning electron microscopy and transmission electron microscopy to study the particle growth mode. The columnar structure of some of the large particles point towards surface growth, while observation of the spherical carbon nanoparticles indicates growth in the plasma phase. Multiple nanoparticles can agglomerate and form bigger particles. The spherical shape of the agglomerates suggests that nanoparticles coalesce in the gas phase. The erosion and re-deposition patterns on the samples are likely determined by the gradients in plasma flux density and surface temperature across the surface.

\footnotetext{
a) Electronic mail: k.bystrov@differ.nl
} 


\section{INTRODUCTION}

Interest in various carbon materials is driven by their unique properties that enable numerous possibilities for industrial applications. In laboratories carbon materials are often synthesized using chemical vapor deposition techniques. redFor example, plasma enhanced chemical vapor deposition (PECVD) is one of the methods to grow carbon nanotubes ${ }^{1}$, amorphous carbon films ${ }^{2}$, diamond films ${ }^{3}$, graphene ${ }^{4}$, carbon nanowalls ${ }^{5}$, etc. Methane, ethylene and acetylene plasmas are commonly used as hydrocarbon sources in conventional PECVD. In the plasma the hydrocarbons can dissociate creating reactive radicals ${ }^{6}$. Generally speaking, the growth process is a competition between deposition of the $\mathrm{CH}_{x}$ radicals and ions and chemical sputtering of carbon from the surface ${ }^{8}$.

The competition between carbon erosion and re-deposition is also a critical issue for fusion devices ${ }^{7}$, where plasma-facing components commonly consist of carbon, because of its very good thermo-mechanical properties. Erosion and re-deposition phenomena can cause plasma contamination with impurity particles, tritium inventory build-up, limited component lifetime, dust formation and material mixing ${ }^{9}$, thus limiting the availability of a reactor.

There is no doubt that the parameters of fusion plasmas (throughout the article we call plasmas with $n_{e} \geq 10^{20} \mathrm{~m}^{-3}$ "high density plasmas" and the associated particle fluxes of the order of $\Gamma_{i} \sim 10^{24} \mathrm{~m}^{-2} \mathrm{~s}^{-1}$ - "high fluxes") differ greatly from those of the PECVD discharges. However, in both cases plasmas consisting of hydrogen, hydrocarbons and sometimes argon are in contact with a carbon surface, inducing etching and, more importantly, deposition. An example of the use of high flux plasma for catalyst-free synthesis of carbon nanotubes and nanowalls ${ }^{10}$ demonstrates that the method might have valuable applications. A problem lies in the lack of understanding of the erosion and re-deposition phenomena under extreme plasma fluxes. Analysis of the "high-flux deposits" and their comparison with the properties of the materials synthesized by conventional PECVD techniques could provide an insight into the mechanisms of deposition under such fluxes.

The high flux plasma conditions can be reproduced in the Pilot-PSI linear plasma generator $^{11}$. As a laboratory device it provides relatively easy diagnostic access, can reach exposure times and accumulated plasma fluencies relevant for fusion reactors, allows easy replacement of exposed samples. Earlier, we have reported formation of large cauliflower-like 
particles on the sample areas exposed to the highest particle flux ${ }^{12-14}$. In this article, we present results of detailed analyses of the formed particles, list possible growth mechanisms and propose an explanation of their presence in the highest flux region.

\section{EXPERIMENTAL}

\section{A. Pilot-PSI and diagnostics}

The Pilot-PSI linear plasma generator is described in detail in ${ }^{15}$. A so-called cascaded arc source $^{16,17}$ creates a plasma, which exhausts into the vacuum vessel along the magnetic field axis. A strong axial magnetic field confines the plasma, generating an intense magnetized cylindrical plasma beam. Pilot-PSI operates in a pulsed mode. The pulse duration is limited by the cooling of the magnetic coils and is a function of the magnetic field strength. Throughout this work the field of $0.4 \mathrm{~T}$ was used. The maximum pulse duration in this case is $160 \mathrm{~s}$. It takes approximately $1 \mathrm{~s}$ for the value of the magnetic field to reach its target value in the beginning of a pulse. The time of ramp down of the B-field at the end of the pulse is approximately $0.5 \mathrm{~s}$. A water-cooled sample holder is located at a distance of 54 $\mathrm{cm}$ from the exit of the plasma source and is installed perpendicularly to the magnetic field. Plasma composition downstream depends on the gas mixture that is supplied to the inlet of the source and the species released from the target. We used hydrogen as the process gas in these experiments.

Plasma electron density $\left(n_{e}\right)$ and electron temperature $\left(T_{e}\right)$ were measured by Thomson scattering at a distance of $\sim 15 \mathrm{~mm}$ upstream from the plasma facing surface ${ }^{18}$. Both $n_{e}$ and $T_{e}$ peak in the middle of the plasma beam and decrease with distance from the axis. Analysis shows that their radial profiles can be fitted with Gaussian curves ${ }^{19}$. The values of $T_{e}$ and $n_{e}$ referred to throughout this article are the peak values. The typical peak ion flux density is $\Gamma_{i} \sim 10^{24} \mathrm{~m}^{-2} \mathrm{~s}^{-1}$, which is approximately two orders of magnitude larger compared to other linear plasma generators ${ }^{20-22}$. Typical full width at half maximum (FWHM) of the plasma beam is $\sim 10 \mathrm{~mm}$. It is important to notice that plasma flux outside FWHM is still $\sim 10^{23} \mathrm{~m}^{-2} \mathrm{~s}^{-1}$ and can not be neglected.

A fast infrared (IR) camera (SC7500-MB, FLIR) was employed to monitor the surface temperature of the samples during exposure. The waveband of the camera spans from 1.5 
$\mu \mathrm{m}$ to $5.1 \mu \mathrm{m}$. 2D surface temperature profiles were measured with a spatial resolution of $0.3 \mathrm{~mm}$. Additionally, surface temperature was monitored by multiwavelength pyrometer (FMPI SpectroPyrometer, FAR associates). The pyrometer integrates the emission spectrum from $1.30 \mu \mathrm{m}$ to $1.65 \mu \mathrm{m}$ and can measure temperatures above $300{ }^{\circ} \mathrm{C}$. Simultaneous use of the IR camera and the pyrometer allows the surface emissivity to be determined by cross-comparison of both techniques. Bulk temperature can be determined by a K-type thermocouple inserted into a sample.

The samples used in this study were machined out of fine-grain graphite (R6650, SGLCarbon). The samples are $4 \mathrm{~mm}$ thick disks with a diameter of $30 \mathrm{~mm}$. Prior to exposure in Pilot-PSI they were mechanically polished and ultrasonically cleaned using acetone and then alcohol. The samples were clamped onto a water cooled copper heat sink. We used flexible Grafoil $^{\circledR}$ sheet as an interface between graphite and copper to improve the thermal contact between the two. The clamping ring was shielding a small part of the sample periphery from plasma impact, so the effective exposed diameter of a sample in this mounting configuration was in fact $26 \mathrm{~mm}$. The energy of the incoming ions was adjusted by negatively biasing the sample with respect to the plasma potential. The minimum ion energy was obtained when the surface was at the floating potential.

A sensitive microbalance was used to determine the mass loss of samples due to plasma exposure. Typical mass loss for a graphite sample exposed to hydrogen plasma for several hundred seconds is in the milligram range, which is two orders of magnitude larger than the sensitivity of the balance. Such mass loss is quite large and makes contributions from absorbed water or hydrogen retention insignificant. Indeed, let us assume that due to exposure to air the graphite surface becomes covered by several monolayers of water. Such amount of water would only weigh approximately $10^{-3} \mathrm{mg}$. As for the deuterium uptake, the experimental conditions are very unfavorable for the retention. It is known that the saturated hydrogen concentration in graphite decreases with temperature and becomes insignificant for temperatures above $1000 \mathrm{~K}^{23}$. Since the temperatures in our experiments were approaching $1500 \mathrm{~K}$, the exposed graphite areas were relatively small and hydrogen atoms are very light we can safely neglect the mass of the retained gas in the measurements. Additional analyses performed after plasma exposure of the samples included surface profilometry, scanning electron microscopy (SEM) and high resolution transmission electron microscopy (HRTEM). 


\section{B. Chemical sputtering measurements in Pilot-PSI}

Generally speaking, removal of carbon from a graphite surface under plasma bombardment falls under the definition of chemical sputtering, i.e. a process where due to ion bombardment a chemical reaction occurs, which produces a particle weakly bound to the surface which then can be desorbed into the gas phase ${ }^{24}$. To avoid confusion between such terms as "chemical erosion" and "chemical sputtering" we have used latter.

The sputtering yield is defined as the ratio between the fluxes of eroded and incoming particles. The incident ion flux in Pilot-PSI is obtained by integrating the ion flux density over the sample surface $S$. According to the sheath theory the ion flux density is a function of electron density $n_{e}$ and electron temperature $T_{e}{ }^{25}$. Assuming that ions in the pre-sheath are accelerated up to the sound velocity and that plasma density in the sheath drops by a factor of 2 compared to the pre-sheath we obtain:

$$
\Gamma_{H^{+}}=\frac{1}{2} \iint_{S} n_{e}(r) \times \sqrt{\frac{2 k T_{e}(r)}{m_{i}}} \mathrm{~d} S .
$$

Here $k$ is the Boltzmann's constant and $m_{i}$ is the mass of a hydrogen ion. We consider only protons when calculating the ion flux since the number of molecular ions is kept low by dissociative recombination into two hydrogen atoms as well as mutual neutralization ${ }^{26}$. One additional assumption is that electron and ion temperatures are equal. This assumption was verified experimentally for the exposure conditions used here ${ }^{26}$. For the negatively biased samples, the ion saturation current measurement was used to cross-check the flux value derived from the Thomson scattering.

During interaction of the low temperature hydrogen ions with carbon the latter can be chemically sputtered. Carbon atoms leave the surface in the form of hydrocarbon molecules ${ }^{27}$. The gross carbon flux leaving the surface can be quantified using optical emission spectroscopy ${ }^{28,29}$. Specifically, molecular CH A-X band emission (Gerö band at 431.42 $\mathrm{nm}$ ) is used as a measure for the eroded $\mathrm{CH}_{4}$ species $^{30}$. Here the $\mathrm{CH} \mathrm{A}-\mathrm{X}$ band emission was recorded by a dedicated spectrometer (wavelength range: $420 \mathrm{~nm}$ - $438 \mathrm{~nm}$, spectral resolution of $\sim 0.1 \mathrm{~nm}$ at $\mathrm{FWHM})^{19,31}$. Inverse photon efficiency $(\mathrm{PE})^{-1}$ was used to convert the $\mathrm{CH}$ photon flux $\phi_{C H}$ into the methane particle flux $\Gamma_{C H_{4}}$ (see Eq. (2)). The inverse photon efficiencies for plasmas with $T_{e}<2 \mathrm{eV}$ have been determined earlier ${ }^{31,32}$.

$$
\Gamma_{C H_{4}}=(P E)^{-1} \times \phi_{C H} .
$$


We then obtain the gross sputtering yield:

$$
Y_{\text {gross }}=\Gamma_{\mathrm{CH}_{4}} / \Gamma_{H^{+}}
$$

With the high plasma density in front of the surface, a significant fraction of the eroded carbon can be re-deposited and then re-eroded again. Indeed, the mean free path for a charge-exchange reaction between a $\mathrm{CH}_{4}$ particle released from the surface and an $\mathrm{H}^{+}$ion is estimated to be less than $1 \mathrm{~mm}$ under Pilot-PSI conditions ${ }^{19}$. Note that electron excitation of the Gerö band becomes inefficient for $T_{e}$ below $1.5 \mathrm{eV}^{32}$. Given that the charge-exchange mean free path is considerably smaller than the width of the plasma beam, the beam is not transparent for eroded methane molecules. On average, $\mathrm{CH}_{4}$ molecules are getting ionized and accelerated back to the surface of the sample before they can escape the plasma beam region. In other words, there is an ongoing recycling of carbon in the described plasmasurface system. This means that the rate at which material is definitely lost from the surface, i.e. the net sputtering rate, is not at all equal to the rate at which carbon leaves the surface (the gross sputtering rate). The net sputtering yield is determined by measuring the mass of the samples before and after exposures. When the mass $\Delta m$ lost by the sample during the exposure is known, the net sputtering yield can be calculated using the following expression:

$$
Y_{n e t}=\frac{N_{A} \times \Delta m / M_{C}}{\Gamma_{H^{+}} \times t} .
$$

Here $N_{A}$ is the Avogadro's constant, $M_{C}$ - molecular mass of carbon and $t$ - total exposure time.

\section{RESULTS AND DISCUSSION}

\section{A. Ion energy influence on sputtering and re-deposition}

Sputtering yields have been measured for different energies $E_{i o n}$ of hydrogen ions striking the surface. The energy $E_{i o n}$ represents the sum of the energy of ions at the entrance of the plasma sheath $\left(E_{s e}\right)$ and the energy, gained during acceleration inside the sheath $\left(E_{a c c}\right)$. According to the Bohm criterion, the ions enter the sheath with at least the sound speed velocity, thus $E_{s e} \sim 2 k T_{e}$ (here, the assumption $T_{e}=T_{i}$ is employed again). For hydrogenic plasmas in contact with the floating samples the voltage drop across the sheath is $\sim 3 k T_{e} / e^{25}$. 
Consequently, in the case of a floating sample $E_{i o n}=E_{s e}+E_{a c c} \sim 2 k T_{e}+3 k T_{e}=5 k T_{e}$. If the sample is biased, the potential drop in the sheath is equal to the difference between the plasma potential $V_{p l}$ and biasing voltage $V_{\text {bias }}$, so $E_{i o n} \sim 2 k T_{e}+e\left(V_{p l}-V_{\text {bias }}\right)$. The plasma potential was determined to be close to $-10 \mathrm{~V}$. For the biasing voltages used in this work the kinetic energy of hydrogen ions is still below the physical sputtering threshold of carbon ${ }^{33}$.

Normally, both gross and net sputtering yields were calculated for a given sample. However, there were a few samples for which only the net loss of carbon has been quantified. Figure ??a shows the evolution of the gross and net sputtering yields of carbon as a function of the impinging ion energy. The threshold energy for chemical sputtering is expected to be in the range of $1-2 \mathrm{eV}$ or lower ${ }^{34}$. By fitting the experimental data we obtain a threshold energy of $1.1 \mathrm{eV}$. The sputtering yield increases by a factor of seven when the ion energy changes from $2.5 \mathrm{eV}$ to $6.5 \mathrm{eV}$. This increase is likely due to the fact that faster ions break more $\mathrm{C}-\mathrm{C}$ bonds within their penetration depth. Thus, more sites for reaction between incoming hydrogen ions with carbon are created, leading to the increased formation rate of the volatile hydrocarbons ${ }^{35}$. For ion energies higher than $10 \mathrm{eV}$, the sputtering yield only increases slightly with the ion energy. Similar behaviour in this ion energy range was observed previously ${ }^{36-38}$.

In our experiments the particle flux to the surface is $\sim 10^{24} \mathrm{~m}^{-2} \mathrm{~s}^{-1}$, which is very high. The corresponding heat flux is of the order of $10 \mathrm{MW} / \mathrm{m}^{2}$. The sample surface temperature is determined by the balance between this incoming heat flux and the cooling capacity of the heat sink. Peak surface temperature of all exposed samples was in the range of $1000{ }^{\circ} \mathrm{C}$ - $1500{ }^{\circ} \mathrm{C}$. Such high temperatures are most certainly measured because of accumulation of re-deposits on the plasma exposed areas. The re-deposits are very poorly attached to the surface. Indeed, in ${ }^{12}$ it was shown that the surface temperature measured by the IR pyrometer increases for every consecutive plasma shot. However, the thermocouple measurements performed later indicate that the temperature in the bulk of the samples remains constant and equal to $250 \pm 20{ }^{\circ} \mathrm{C}$. This points out to strong plasma-induced surface morphology changes.

We define the local re-deposition fraction $\alpha$ as the fraction of carbon re-deposited on the sample surface and the gross amount of eroded carbon:

$$
\alpha=\frac{Y_{\text {gross }}-Y_{\text {net }}}{Y_{\text {gross }}} \times 100 \%
$$


Using this definition, $\alpha$ was calculated for those samples for which both gross and net sputtering yields were measured (see Figure ??b). Interestingly, a strong increase of the redeposition fraction is observed for energies in the range of $2-60 \mathrm{eV}$. Up to $90 \%$ of the eroded particles are actually re-deposited on the sample surface. In other words, the net erosion is reduced by a factor of ten with respect to the gross erosion. The increase of the gross erosion with the ion energy for a given ion flux, effectively means an increase of the hydrocarbon flux returning to the surface. Hence the carbon-to-hydrogen flux ratio increases with the ion energy favouring re-deposition of the material on the surface ${ }^{39}$. However, it obviously does not prevent the net loss of material from the sample. Indeed, $Y_{\text {net }}$ is approximately 0.01 for energies below $10 \mathrm{eV}$ and this value doesn't decrease for larger values of $E_{\text {ion }}$.

\section{B. Location and structure of the re-deposits}

Stylus profiling (Veeco Dektak 6M Stylus Profiler) was used to study the evolution of the surface profile after the plasma exposure. An example of the surface profile is presented in Figure ??a. The reference zero level of the profile is determined by the edges of the sample which were shielded from the plasma. The main feature of such profile is noticeable immediately - the re-deposited material accumulates in the center of the sample, where the plasma flux reaches its peak value.

The fine structure of the re-deposit in Pilot-PSI as observed by SEM was first reported $\mathrm{in}^{12}$. The deposit appears in the form of microparticles (Figure ??b). X-ray photoelectron spectroscopy and energy dispersive X-ray spectroscopy confirm that the microparticles consist solely of carbon. At the same time peripheral regions of the samples become rough and feature spiked structures, as shown in Figure ??c. Such morphology is characteristic for graphite chemically sputtered by hydrogen isotopes. For instance, similar spiked structures were observed on PISCES-B ${ }^{40}$ and in ion beam experiments ${ }^{41}$.

The observed microparticles could be provisionally divided into three categories based

on their appearance and structure. The first type consists of symmetric particles with a rough surface, as shown in Figure ??a. The surface is formed by what appears to be a conglomeration of folded graphitic sheets. Microparticles of the second type also have rough surfaces, however they are clearly not symmetric (Figure ??b). One explanation of such shape is the presence of the prevailing growth direction. Finally, the third type of particles 
is characterized by a much smoother surface (Figure ??c) compared to the first two types.

Such simplifying classification allows us to note some tendencies in the appearance of the deposited particles. For instance, particles found in the very center of the samples, i.e. in the center of the plasma beam, are generally smoother than those found a few millimeters off center. Also, type II particles are usually observed on the edges of deposition region, with their elongated sides pointing towards the middle of the plasma beam. Figure ?? shows conglomerates of rough and smooth microparticles observed at the same location. Although this situation is not common, it indicates that different formation mechanisms might take place locally at the same time.

Similar structures were formed previously in linear plasma simulator experiments ${ }^{42,43}$, using high-pressure inductively-coupled plasmas $^{44}$, helicon-wave excited discharge ${ }^{45}$ and even in the tokamak environment ${ }^{46,47}$. Furthermore, analogous structures were observed in PECVD processing. For instance - during early stages of ultrananocrystalline diamond growth $^{48,49}$ or during growth of carbon nanowalls ${ }^{5,50}$. The exposures in Pilot-PSI stand out since they were performed at plasma flux at least two orders of magnitude higher than in the other experiments listed here. The similarities between the structures, as observed by SEM, are remarkable given the differences in the plasma parameters.

\section{Influence of plasma conditions on the deposited microparticles}

The influence of the plasma conditions on microparticle populations was studied. For this purpose, SEM images of the areas subjected to peak particle flux (and most densely covered by deposits) were analyzed for samples exposed to various conditions. For each image, the number of particles, their size and surface coverage were evaluated. The results are presented in the form of histograms in Figure ??.

For samples exposed at $T_{e}=0.5 \mathrm{eV}$ the mean size of the formed microparticles is roughly $10 \mu \mathrm{m}$. There are hardly any particles larger than $20 \mu \mathrm{m}$ on the surface. Increase of the incident energy of hydrogen ions triggers the formation of larger particles. The particles reach $\sim 60 \mu \mathrm{m}$ in diameter. At the same time the surface becomes fully covered by the re-deposit and particles pile up on top of each other. The surface coverage is plotted against the fraction of re-deposited carbon in Figure ??. Another interesing feature is the seeming presence of two particle populations for the case of the highest ion energy. The respective mean sizes 
of the particles from the two populations are $\sim 15 \mu \mathrm{m}$ and $\sim 35 \mu \mathrm{m}$. The reproducible presence of two groups of carbon particles was reported $\mathrm{in}^{51}$, where they grew in a cathode sputtering discharge. The authors assumed that the larger population could be produced during the plasma extinction phase by agglomeration of the smaller particles. Note that we have checked that the $0.5 \mathrm{~s}$ ramp down of the magnetic field in the end of the Pilot-PSI discharge as opposed to the instantaneous termination of the plasma does not influence the size distribution of the particle population.

Note that the particles illustrated in Figure ?? were formed after approximately $200 \mathrm{~s}$ of plasma exposure in Pilot-PSI, which corresponds to an ion fluence of $\sim 10^{26} \mathrm{~m}^{-2}$. This means that the growth rate for the biggest microparticles can be estimated at $\sim 0.15 \mu \mathrm{m} / \mathrm{s}$. Growth dynamics of the microparticles during the first $100 \mathrm{~s}$ of the plasma exposure is illustrated in Figure ??. A gradual coverage of the surface with particles is observed. It remains to be seen whether particle growth and accumulation saturate, as an attempted exposure of 1000 s resulted in a loss of particles from the surface, apparently due to flaking.

An insight into particle growth mechanisms can be obtained from analysis of their internal structure. Figure ?? shows a type II particle which was probably broken during handling and transportation of the sample. Note that the particle is actually not spherical, but has a hemispherical shape. Such a shape unambiguously points to the fact that growth of this particle occured on the surface. Indeed, the sheath thickness in Pilot-PSI is of the order of several microns. So, already very small imperfections on the surface distort the plasma sheath, directing incoming ions towards the growth site. A hemispherical particle grows because the depositing species arrive from all directions. Columnar structures can be distinguished in the magnified image of the particle cross-section, supporting the argument of growth on the surface for this type of the re-deposits.

Several individual particles were isolated for focused ion beam milling. Up to now we were able to analyze only type III particles (symmetric particles with smooth surfaces) with this method. The SEM images reveal that the particles consist of disordered graphitic planes which do not show any preferrential orientation (see Figure ??). There are many voids inside the particle. The periphery consists of graphitic "ribbons", organized in arches (Figure ??b). It is crucial to point out that the particle shown here does not seem to be connected with the underlying surface, but rather lies on top of it.

The material from the inside of the spherical microparticles was studied by HRTEM. 
Many carbon nanoparticles have been observed inside graphitic layers which form the microparticles. A number of those particles are shown in Figure ??a. The particles are smaller than $10 \mathrm{~nm}$ in diameter. $\mathrm{In}^{52}$ it was shown that such nanoparticles can be grown in sputtering discharges. Moreover, large quantities of similar nanoparticles have been found in Mega Ampere Spherical Tokamak (MAST) and Tore Supra tokamak ${ }^{52-54}$. The onion-like shape of the particles suggests that they were grown in the plasma.

Figure ??b shows several nanoparticles, which have coalesced. The size of the agglomerated particle is around $0.2 \mu \mathrm{m}$. Concentric nature of the graphitic sheets on the periphery suggests that the primary particles have agglomerated in the plasma. The agglomeration phase was followed by a growth phase by carbon deposition. In the latter case, carbon atoms are arranged in graphitic layers as shown in Figure ??c. Two-stage growth of similar carbon nanoparticles has already been observed in low pressure, low input power plasmas ${ }^{55}$, however graphitization is more pronounced and the particles are larger in present experiments. Clarification of the exact growth mechanism will be the subject of future work. Taking into account the residence time of neutrals in the plasma beam and known reaction rates, it appears unlikely that the growth occurs through neutral-neutral interactions. On the other hand, the plasma potential profile allows negative ions to be confined in the plasma beam and ion-driven chemistry might promote the particle growth ${ }^{56,57}$.

\section{DISCUSSION}

One particular interesting outcome of these experiments is agglomeration of re-deposits in the region of peak particle and plasma flux. At first sight this observation appears counterintuitive. In this section we attempt to explain the existence of the observed erosion and re-deposition patterns. Spatial variation of the carbon sputtering yield across the surface of the samples and transport of the eroded hydrocarbons are taken into account.

An extensive analytical model for the chemical sputtering of carbon based materials by hydrogen ions as a function of the ion impact energy, surface temperature and the ion flux has been developed by Roth and co-authors ${ }^{58,59}$. The model relies on a collection of experimental results at ion fluxes below $10^{24} \mathrm{~m}^{-2} \mathrm{~s}^{-1}$ and predicts a monotonic decrease of the sputtering yield with the increasing flux. In Pilot-PSI the plasma flux density peaks in the center of the beam and decays towards the edges. An example of the calculated flux 
density profile is plotted in Figure ??b. The shape of the plasma beam dictates the heat flux distribution across the exposed surface of a sample. Naturally, the surface temperature profile also peaks in the center, with the edges being several hundreds degrees cooler. Such a profile, as measured with the IR camera is shown in Figure ??a.

Using the measurements of the plasma flux and the surface temperature as an input, we calculate the gross sputtering yield (Figure ??c) and the carbon flux into the plasma (Figure ??d) according to the equations given in ${ }^{58}$ and $^{59}$. Due to low hydrogen impact energies the total sputtering yield $Y_{\text {tot }}$ (here and further on we use the notations from ${ }^{58}$ ) is dominated by the thermal erosion yield $Y_{\text {therm }}$, while the physical sputtering, radiation damage effects and the sputtering of radicals are negligible. Note, that when $Y_{\text {tot }}$ is calculated the flux dependence is not taken into account. The total sputtering yield $Y_{\text {Roth }}$ is plotted separately to discriminate the effect the flux profile has on the sputtering yield.

The surface temperature distribution plays a key role in shaping the radial profile of the sputtering yield. The yield peaks approximately $5 \mathrm{~mm}$ away from the center of the sample due to the favourable temperature conditions. This effect is further amplified by the tendency of the higher flux to decrease the yield. In general, the lower sputtering yield does not necessarily translate into the lower number of eroded species. However, in this experiment it is the case. The highest local influx of carbon into the plasma is from the net erosion region, where the sputtering yield peaks. This is easy to see if one compares the profiles in (Figure ??c) and (Figure ??d). According to the calculation the carbon flux into the plasma from the net erosion region exceeds the flux from the central region by almost three orders of magnitude.

We are now ready to propose a possible qualitative explanation of the accumulation of the re-deposited carbon in the region of the peak particle flux. Due to the temperature and flux gradients across the surface the center of the sample is effectively a trap for re-deposited carbon. The net erosion region acts as a supplier of carbon. We can assume the cosine angular destribution of velocities of the hydrocarbon molecules leaving the surface ${ }^{60}$. Thus, a fraction of the sputtered species travels towards the center of the plasma beam. They quickly become ionized and driven towards the surface. Once re-deposited, the probability for a given carbon atom to be eroded again is significantly less than originally, because of the high surface temperature and the high plasma flux. In other words, the incoming flux of carbon from the net erosion region is large enough, and the sputtering yield in the centre 
of the sample is small enough to trigger accumulation of the re-deposits and formation of microparticles.

Such explanation is only qualitative since the numerical values of the chemical sputtering yield predicted by the model are approximately two orders of magnitude lower then the measured ones. Rapid surface modification could be one of the factors contributing to this discrepancy. On one hand, physical and chemical properties of the re-deposits, e.g. density, porosity, chemical bond strength, are not identical to the properties of an initial graphite surface. The dependence of the yield on structural properties of the material is particularly high at very low impact energy ${ }^{27,61}$, which is exactly the case in this study. Moreover, simulations show that re-deposited species suffer from enhanced re-erosion at plasma-wetted areas under simultaneous ion bombarding ${ }^{62}$. Specifically, re-erosion of re-deposited species is enhanced by a factor of 5-10 compared to the erosion of the substrate material. On the other hand, strong roughening of the surface observed with electron microscopy leads to the increase of the effective surface area seen by the plasma. Consequently, the effective plasma flux at the surface is smaller than the value calculated using Thomson scattering. However, due to wide variety of the morphologies on the surface and their evolution during a given exposure it is difficult to quantify this effect reliably.

\section{CONCLUSIONS}

Exposure of graphite samples to high density low temperature hydrogen plasma results in the release of hydrocarbons from the surface due to chemical sputtering. The gross sputtering yield increases sharply with the impinging ion energy in the region $2.5 \mathrm{eV}<E_{\text {ion }}<6.5$ $\mathrm{eV}$ and then increases only slightly for energies above $10 \mathrm{eV}$. The short mean free path of charge-exchange recombination makes the plasma beam opaque for the released hydrocarbon molecules. On average, up to $90 \%$ of eroded hydrocarbons are re-deposited on the surface. The samples are covered with carbon microparticles, that are concentrated in the area of highest heat and particle flux. The morphology of the particles as observed by SEM is similar to some examples from the conventional PECVD processing. The re-deposition in the center of the samples is probably driven by the gradients of the surface temperature and the plasma flux across the surface. The largest particles are observed for the highest re-deposition rate with an estimated growth rate of up to $0.15 \mu \mathrm{m} / \mathrm{s}$. The particles can 
be divided into categories based on their appearance. Differences in shape and internal structure imply that various types of particles grow by different mechanisms. Some of the microparticles are hemispherical with columnar-like internal structure, indicating a surface growth. Additionally, dense spherical particles consisting of disordered graphitic planes with embedded carbon nanoparticles are observed. Carbon nanoparticles can agglomerate in the plasma phase. Such agglomerates continue to grow by carbon deposition.

In general, the formation of carbon particles on substrates exposed to high flux plasmas demonstrates that such plasmas can potentially be used for surface structuring and carbon material synthesis on micro- and nanoscale.

\section{ACKNOWLEDGMENTS}

This work was supported by the European Communities under the Contract of Association between EURATOM/FOM and carried out within the framework of the European Fusion Programme with financial support from NWO and the NWO Grant RFBR 047.018.002. This work was also made within the framework of EFDA and a contract of the French FR FCM - EURATOM/CEA association. The views and opinions expressed herein do not necessarily reflect those of the European Commission.

\section{REFERENCES}

${ }^{1}$ S. J. Chung, S. H. Lim, C. H. Lee, and J. Jang, Diam. Relat. Mater. 10(2), 248 (2001).

${ }^{2}$ G. Capote, R. Prioli, P. M. Jardim, A. R. Zanatta, L. G. Jacobsohn, and F. L. Freire Jr., J. Non-Cryst. Solids 338-340 (1S), 503 (2004).

${ }^{3}$ G. Fedosenko, A. Schwabedissen, J. Engemann, E. Braca, L. Valentini, and J. M. Kenny, Diam. Relat. Mater. 11 (3-6), 1047 (2002).

${ }^{4}$ J. L. Qi, W. T. Zheng, X. H. Zheng, X. Wang, and H. W. Tian, Appl. Surf. Sci. 257 (15), $6531(2011)$.

${ }^{5}$ A. T. H. Chuang, B. O. Boskovic, and J. Robertson, Diam. Relat. Mater. 15, 1103 (2006).

${ }^{6}$ M. Meyyappan, L. Delzeit, A. Cassell, and D. Hash, Plasma Sources Sci. T. 12(3), 205 (2003).

${ }^{7}$ W. Jacob, J. Nucl. Mater. 337-339, 839 (2005). 
${ }^{8}$ A. von Keudell and W. Jacob, J. Appl. Phys. 79(2), 1092 (1996).

${ }^{9}$ G. Federici, P. Andrew, P. Barabaschi, J. Brooks, R. Doerner, A. Geier, et al., J. Nucl. Mater. 313-316, 11 (2003).

${ }^{10}$ F. Liu, K. Bystrov, G. De Temmerman, L. Xu, and X. Li, Submitted to Carbon.

${ }^{11}$ J. Westerhout, W. R. Koppers, W. A. J. Vijvers, R. S. Al, S. Brezinsek, S. Brons, et al., Phys. Scripta T128, 18 (2007).

${ }^{12}$ K. Bystrov, J. Westerhout, M. Matveeva, A. Litnovsky, L. Marot, E. Zoethout, et al., J. Nucl. Mater. 415(1,Supplement), S149 (2011).

${ }^{13}$ G. M. Wright, J. Westerhout, R. S. Al, E. Alves, L. C. Alves, N. P. Barradas, et al., J. Nucl. Mater. 417(1-3), 457 (2011).

${ }^{14} \mathrm{~J}$. Westerhout, Ph.D. thesis, Carbon chemical erosion in high flux and low temperature hydrogen plasma (Eindhoven, The Netherlands, 2010).

${ }^{15}$ G. J. van Rooij, V. P. Veremiyenko, W. J. Goedheer, B. de Groot, A. W. Kleyn, P. H. M. Smeets, et al., Appl. Phys. Lett. 90(12), 121501 (2007).

${ }^{16}$ G. M. W. Kroesen, D. C. Schram, and J. C. M. de Haas, Plasma Chem. Plasma P. 10, 531 (1990).

${ }^{17}$ W. A. J. Vijvers, C. A. J. van Gils, W. J. Goedheer, H. J. van der Meiden, D. C. Schram, V. P. Veremiyenko, et al., Phys. Plasmas 15(9), 093507 (2008).

${ }^{18}$ H. J. van der Meiden, R. S. Al, C. J. Barth, A. J. H. Donné, R. Engeln, W. J. Goedheer, et al., Rev. Sci. Instrum. 79(1), 013505 (2008).

${ }^{19}$ J. Westerhout, D. Borodin, S. Brezinsek, N. J. Lopes Cardozo, J. Rapp, D. C. Schram, et al., Nucl. Fusion 50(9), 095003 (2010).

${ }^{20}$ B. Unterberg, R. Jaspers, R. Koch, V. Massaut, J. Rapp, D. Reiter, et al., Fusion Eng. Des. 86(9-11), 1797 (2011).

${ }^{21}$ A. Kreter, Fusion Sci. Tech. 59(1T), 51 (2011).

${ }^{22}$ G. J. van Rooij, Fusion Sci. Tech. 61(2T), 266 (2012).

${ }^{23}$ T. Tanabe, N. Bekris, P. Coad, C. H. Skinner, M. Glugla, and N. Miya, J. Nucl. Mater. 313-316, 478 (2003).

${ }^{24}$ A. Kirschner, Fusion Sci. Tech. 61(2T), 230 (2012).

${ }^{25}$ P. C. Stangeby, The Plasma Boundary of Magnetic Fusion Devices (Institute of Physics Publishing, Bristol and Philadelphia, 2000), pp. 61-110.

${ }^{26}$ A. E. Shumack, V. P. Veremiyenko, D. C. Schram, H. J. de Blank, W. J. Goedheer, H. J. 
van der Meiden, et al., Phys. Rev. E 78, 046405 (2008).

${ }^{27}$ W. Jacob and J. Roth, Chemical Sputtering. In: R. Behrisch, W. Eckstein, editors. Sputtering by Particle Bombardment (Springer, Berlin Heidelberg New York, 2007), pp. 329400 .

${ }^{28}$ S. Brezinsek, A. Pospieszczyk, A. Kirschner, G. Sergienko, A. Huber, V. Philipps, et al., Phys. Scripta T111, 42 (2004).

${ }^{29}$ D. G. Whyte, J. N. Brooks, P. C. Stangeby, and N. H. Brooks, Phys. Scripta T111, 34 (2004).

${ }^{30}$ K. Behringer, J. Nucl. Mater. 176-177, 606 (1990).

${ }^{31}$ J. Westerhout, N. J. Lopes Cardozo, J. Rapp, and G. J. van Rooij, Appl. Phys. Lett. 95(15), 151501 (2009).

${ }^{32}$ G. A. van Swaaij, K. Bystrov, D. Borodin, A. Kirschner, L. B. van der Vegt, G. J. van Rooij, et al., Plasma Phys. Contr. F. 54, 095013 (2012)

${ }^{33}$ W. Eckstein, Sputtering Yields. In: R. Behrisch, W. Eckstein, editors. Sputtering by Particle Bombardment (Springer, Berlin Heidelberg New York, 2007), pp. 33-187.

${ }^{34}$ J. Roth and C. García-Rosales, Nucl. Fusion 36(12), 1647 (1996).

${ }^{35}$ C. Hopf and W. Jacob, J. Nucl. Mater. 342(1-3), 141 (2005).

${ }^{36}$ B. V. Mech, A. A. Haasz, and J. W. Davis, J. Nucl. Mater. 241-243, 1147 (1997).

${ }^{37}$ B. V. Mech, A. A. Haasz, and J. W. Davis, J. Nucl. Mater. 255(2-3), 153 (1998).

${ }^{38}$ M. Balden and J. Roth, J. Nucl. Mater. 280(1), 39 (2000).

${ }^{39}$ D. Naujoks amd W. Eckstein, J. Nucl. Mater. 220-222, 993 (1995).

${ }^{40}$ R. Pugno, M. J. Baldwin, R. P. Doerner, J. Hanna, D. Nishijima, and G. Antar, J. Nucl. Mater. 363, 1277 (2007).

${ }^{41}$ H. Zhang, F. W. Meyer, H. M. Meyer III, and M. J. Lance, Vacuum 82(11), 1285 (2008).

${ }^{42}$ N. Ohno, Y. Kobayashi, T. Sugimoto, and S. Takamura, J. Nucl. Mater. 337(1-3), 35 (2005).

${ }^{43}$ N. Ohno, M. Yoshimi, M. Tokitani, S. Takamura, K. Tokunaga, and N. Yoshida, J. Nucl. Mater. 390-391, 61 (2009).

${ }^{44}$ Y. Takeguchi, M. Kyo, Y. Uesugi, Y. Tanaka, and S. Masuzaki, Phys. Scripta T138, 014056 (2009).

${ }^{45}$ K. Sasaki, T. Maeda, N. Takada, M. Aramaki, M. Goto, S. Muto, et al., Jpn. J. Appl. Phys. 1 44(10), 7614 (2005). 
${ }^{46}$ N. Asakura, T. Hayashi, N. Ashikawa, T. Hatae, and T. Nakano, Fusion Sci. Technol. 60(4), 1572 (2011).

${ }^{47}$ V. I. Krauz, Yu. V. Martynenko, N. Yu. Svechnikov, V. P. Smirnov, V. G. Stankevich, and L. N. Khimchenko, Physics - Uspekhi 53(10), 1015 (2010).

${ }^{48}$ N. N. Naguib, J. W. Elam, J. Birrell, D. S. Grierson, J. Wang, B. Kabius, et al., Chem. Phys. Lett. 430(4-6), 345 (2006).

${ }^{49}$ O. A. Williams, Diam. Relat. Mater. 20(5-6), 621 (2011).

${ }^{50}$ Z. Bo, K. Yu, G. Lu, P. Wang, S. Mao, and J. Chen, Carbon 49(6), 1849 (2011).

${ }^{51}$ C. Dominique and C. Arnas, J. Appl. Phys. 101(12), 123304 (2007).

${ }^{52}$ C. Arnas, C. Martin, P. Roubin, B. Pégourié, G. De Temmerman, K. Hassouni, et al., Plasma Phys. Contr. F. 52(12), 124007 (2010).

${ }^{53}$ C. Arnas, C. Pardanaud, C. Martin, P. Roubin, G. De Temmerman, and G. Counsell, J. Nucl. Mater. 401(1-3), 130 (2010).

${ }^{54}$ M. Richou, C. Martin, P. Delhaes, M. Couzi, W. Saikaly, C. Brosset, et al., Carbon 45(14), 2723 (2007).

${ }^{55}$ C. Arnas, A. Mouberi, K. Hassouni, A. Michau, G. Lombardi, X. Bonnin, et al., J. Nucl. Mater. 390-391, 140 (2009).

${ }^{56}$ A. A. Howling, L. Sansonnens, J.-L. Dorier, and Ch. Hollenstein, J. Appl. Phys. 75(3), 1340 (1994).

${ }^{57}$ A. Michau, G. Lombardi, C. Arnas, L. C. Delacqua, M. Redolfi, X. Bonnin, et al., Plasma Sources Sci. T. 19(3), 034023 (2010).

${ }^{58}$ J. Roth, J. Nucl. Mater. 266-269, 51 (1999).

${ }^{59}$ J. Roth, A. Kirschner, W. Bohmeyer, S. Brezinsek, A. Cambe, E. Casarotto, et al., J. Nucl. Mater. 337-339, 970 (2005).

${ }^{60}$ J. Marian, L. A. Zepeda-Ruiz, N. Couto, E. M. Bringa, G. H. Gilmer, P. C. Stangeby, et al., J. Appl. Phys. 101, 044506 (2007).

${ }^{61}$ E. Vietzke and V. Phillips, Fusion Technol. 15(1), 108 (1989).

${ }^{62}$ A. Kirschner, A. Kreter, P. Wienhold, S. Brezinsek, J. W. Coenen, H. G. Esser, et al., J. Nucl. Mater. 415(1,Supplement), S239 (2011). 


\section{FIGURE CAPTIONS}

Fig. 1. Gross and net carbon erosion yields as functions of incident hydrogen ion energy. (b) Local re-deposition fraction as a function of incident hydrogen ion energy.

Fig. 2. An example of surface profilometry measurement illustrating accumulation of re-deposited material in the central region of the sample. (b) SEM images of areas covered with deposits as well as deposit-free surfaces, which were experiencing net erosion (c). Here images of different samples are presented to show diversity of possible morphologies.

Fig. 3. SEM images of microparticles of different types: (a) - spherical, symmetric particles; (b) - a particle showing prevailing growth direction; (c) - particles with smooth surface.

Fig. 4. Conglomerates of two different types of microparticles localized in the same place.

Fig. 5. SEM images of microparticles observed at different incident ion energies and corresponding size histograms. Significant amount of larger particles is formed when the target is biased negatively.

Fig. 6. Fraction of the substrate surface covered by the microparticles as a function of the re-deposition efficiency.

Fig. 7. SEM images of the sample surface after $5 \mathrm{~s}, 30 \mathrm{~s}$ and $100 \mathrm{~s}$ of exposure to hydrogen plasma.

Fig. 8. SEM images of a hemispherical particle which is broken in half. The particle shape and columnar structures (seen in the encircled region on the right) indicate surface growth.

Fig. 9. SEM image of a microparticle cut with focused ion beam. 1 - the core, consisting of disordered graphitic planes lacking preferential orientation, 2 - the periphery formed by graphitic "ribbons". (b) TEM image of the "ribbons", organized in arches.

Fig. 10. HRTEM micrographs of spherical nanoparticles (encircled) trapped inside graphitic layers; (b) an agglomeration of nanoparticles forming a core of a bigger particle; (c) concentric graphitic layers on the periphery of the bigger nanoparticle.

Fig. 11. (a) Surface temperature profiles as measured by the IR camera. (b) Plasma flux density profile reconstructed using the Thomson scattering measurements. (c) The chemical sputtering yields calculated in accordance with the Roth model. The difference between $Y_{t o t}$ and $Y_{\text {Roth }}$ is that the flux dependence of the sputtering yield is taken into account in the 
latter. (d) The profile of the carbon influx into the plasma. 


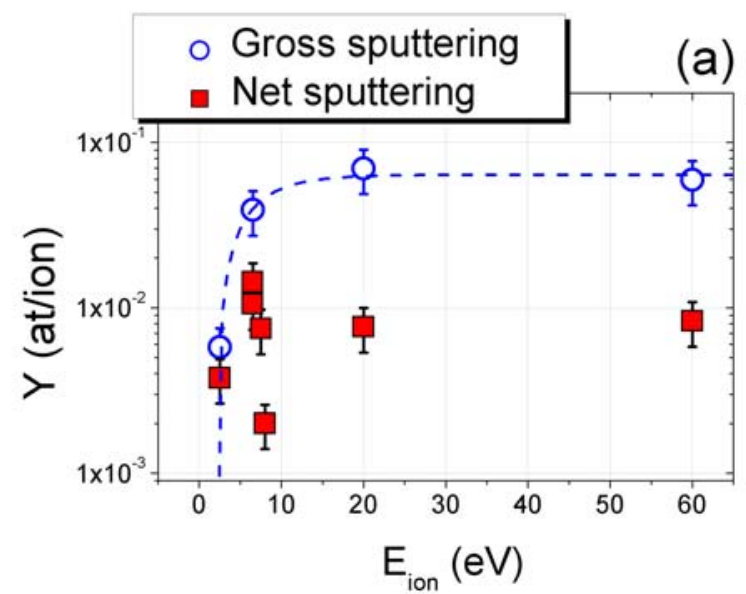

(b)

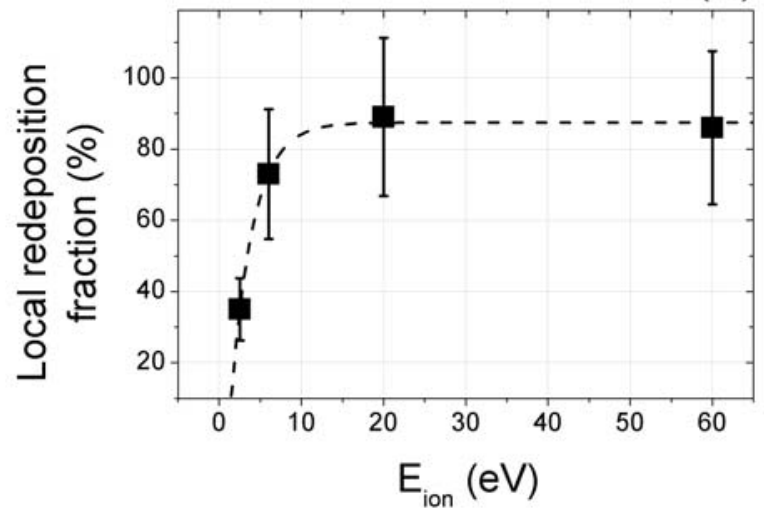



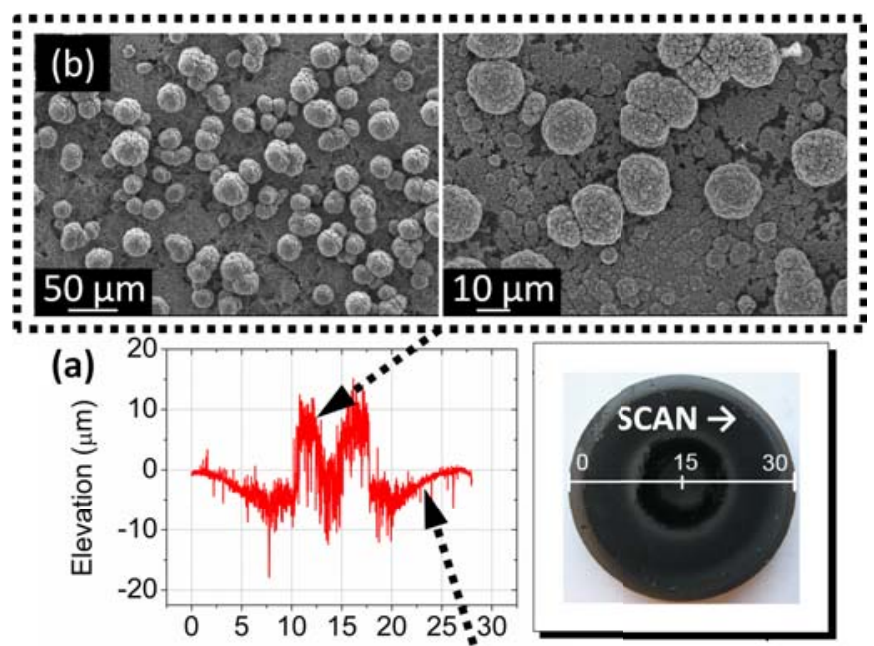
Scan length $(\mathrm{mm})$

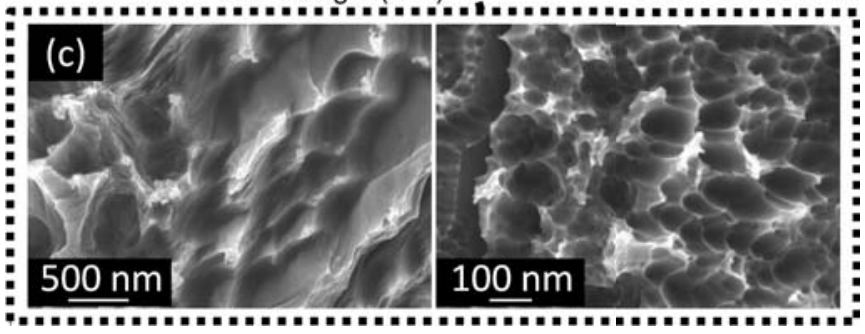




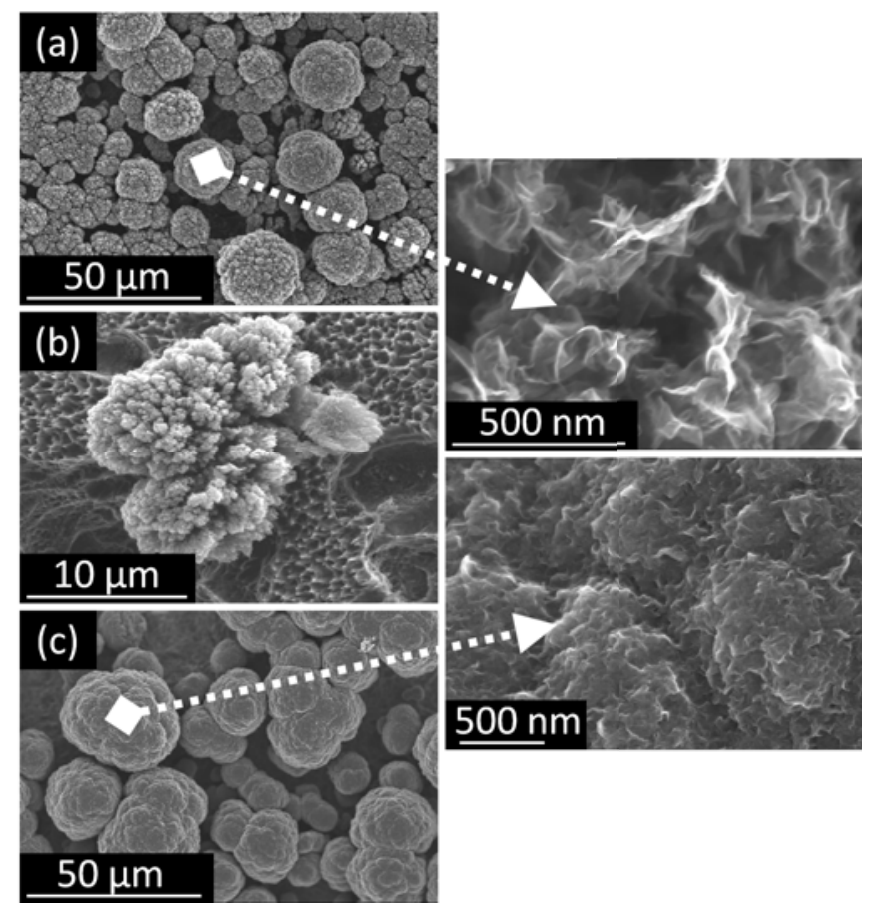




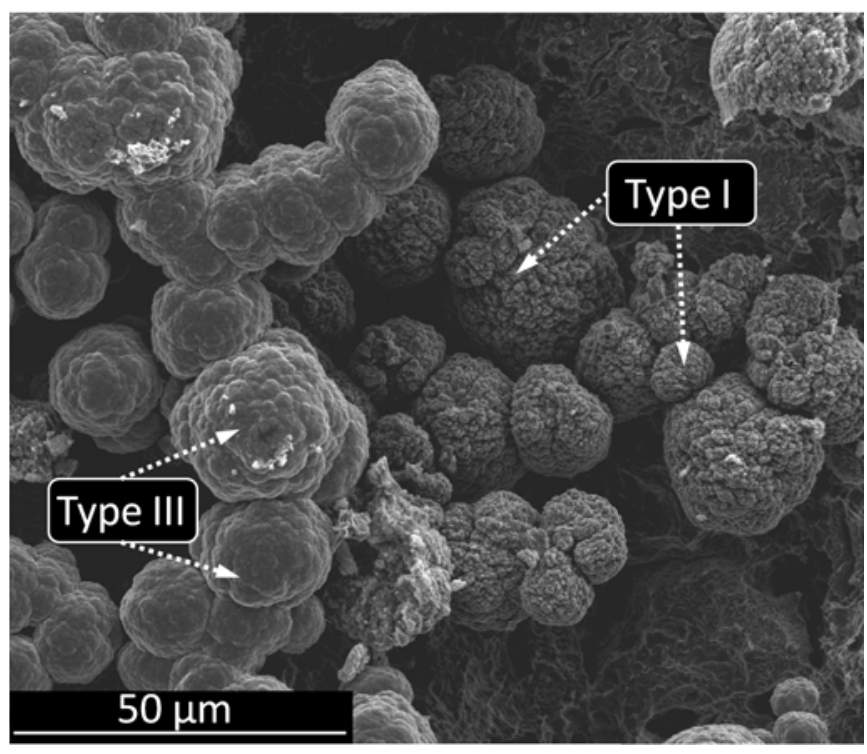



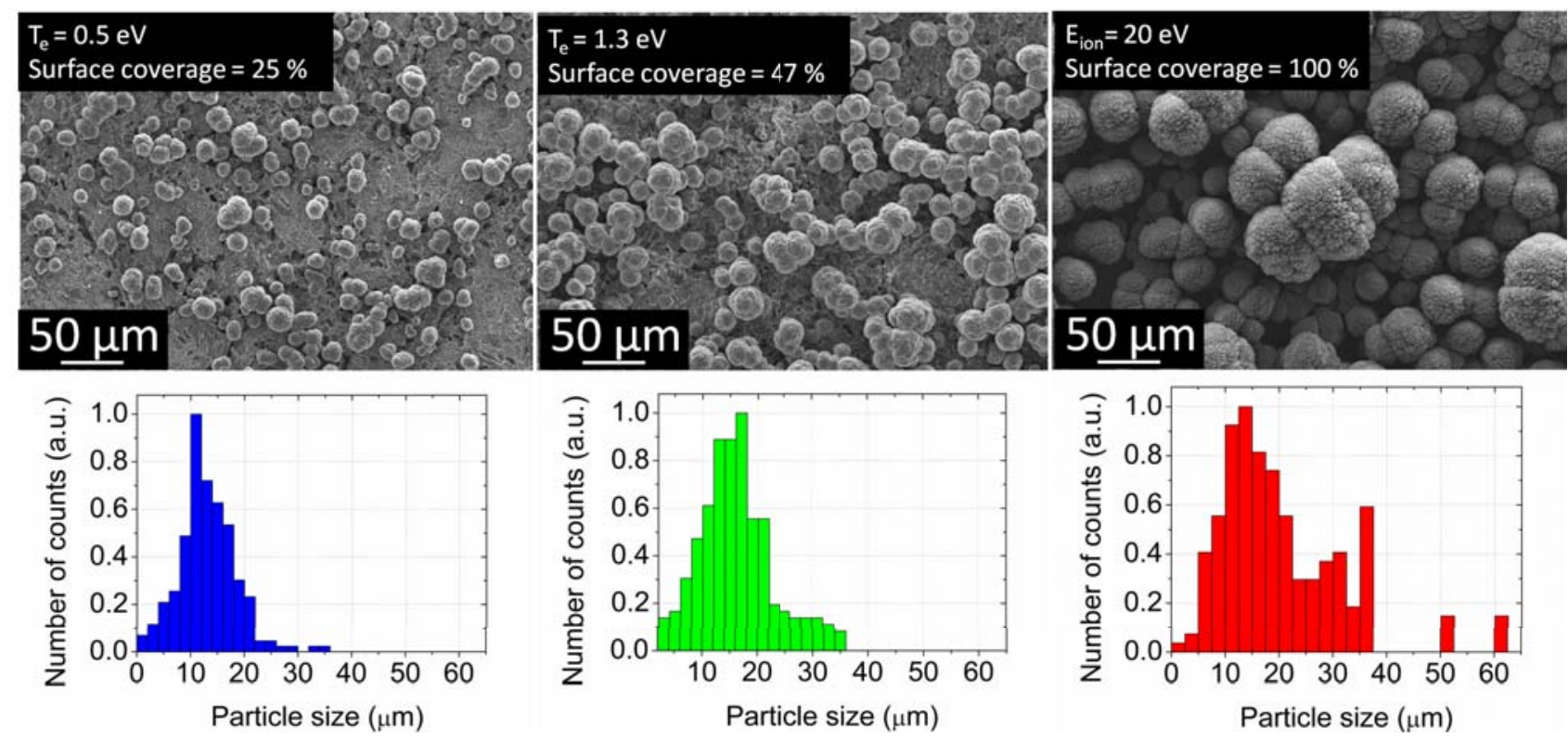


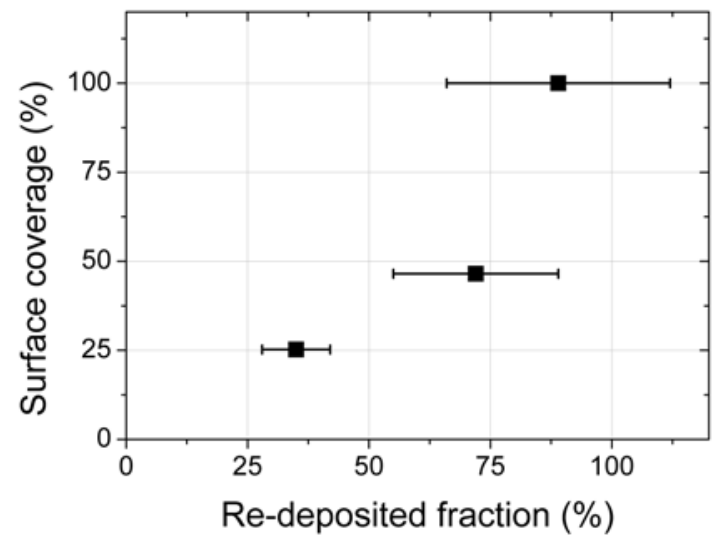



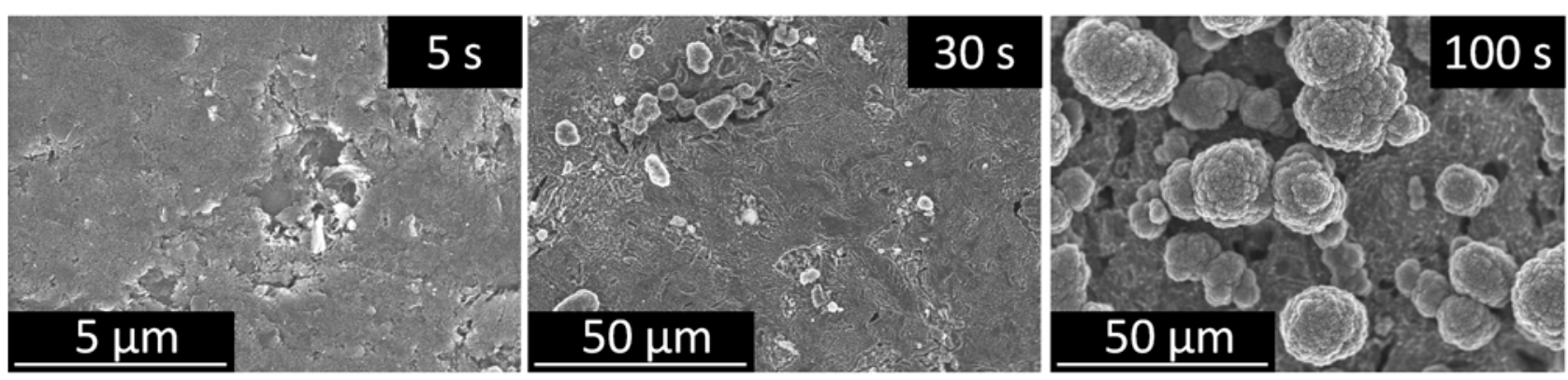

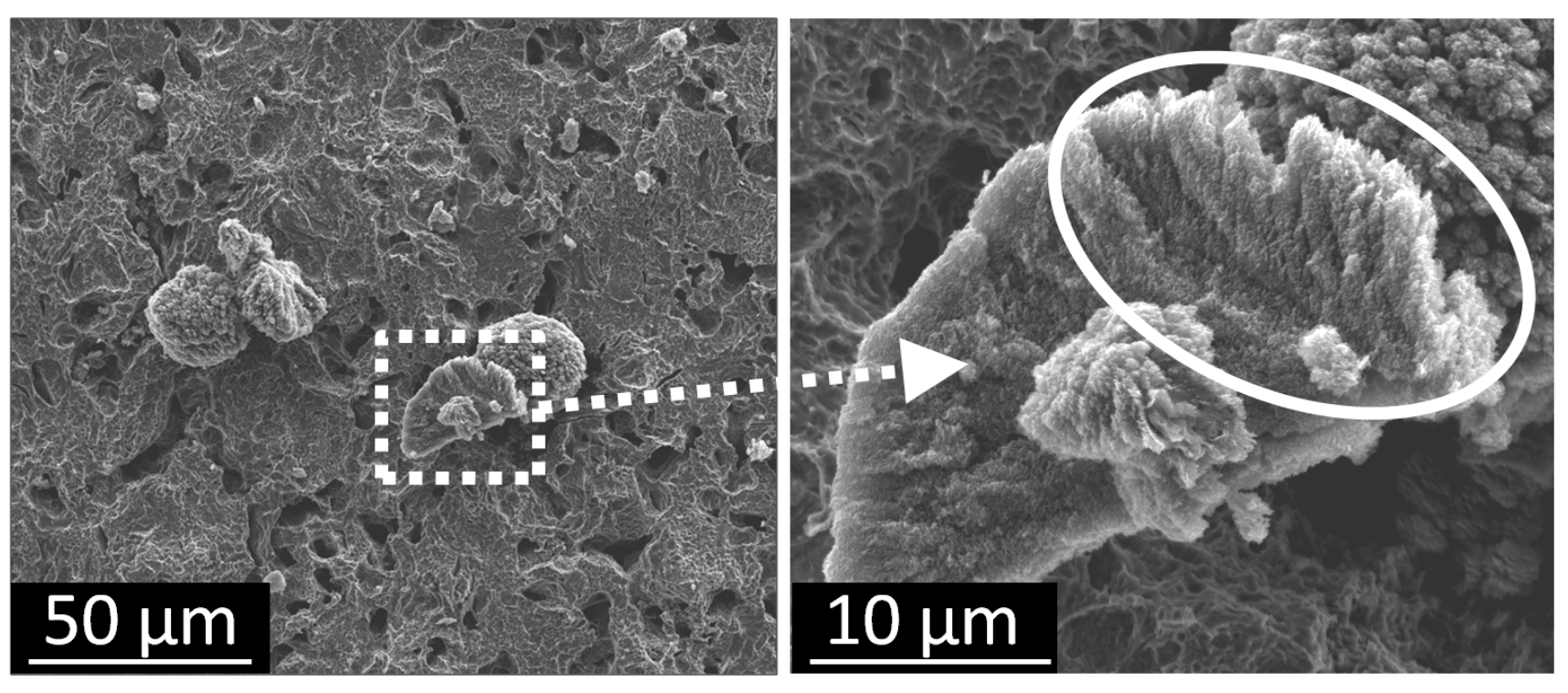


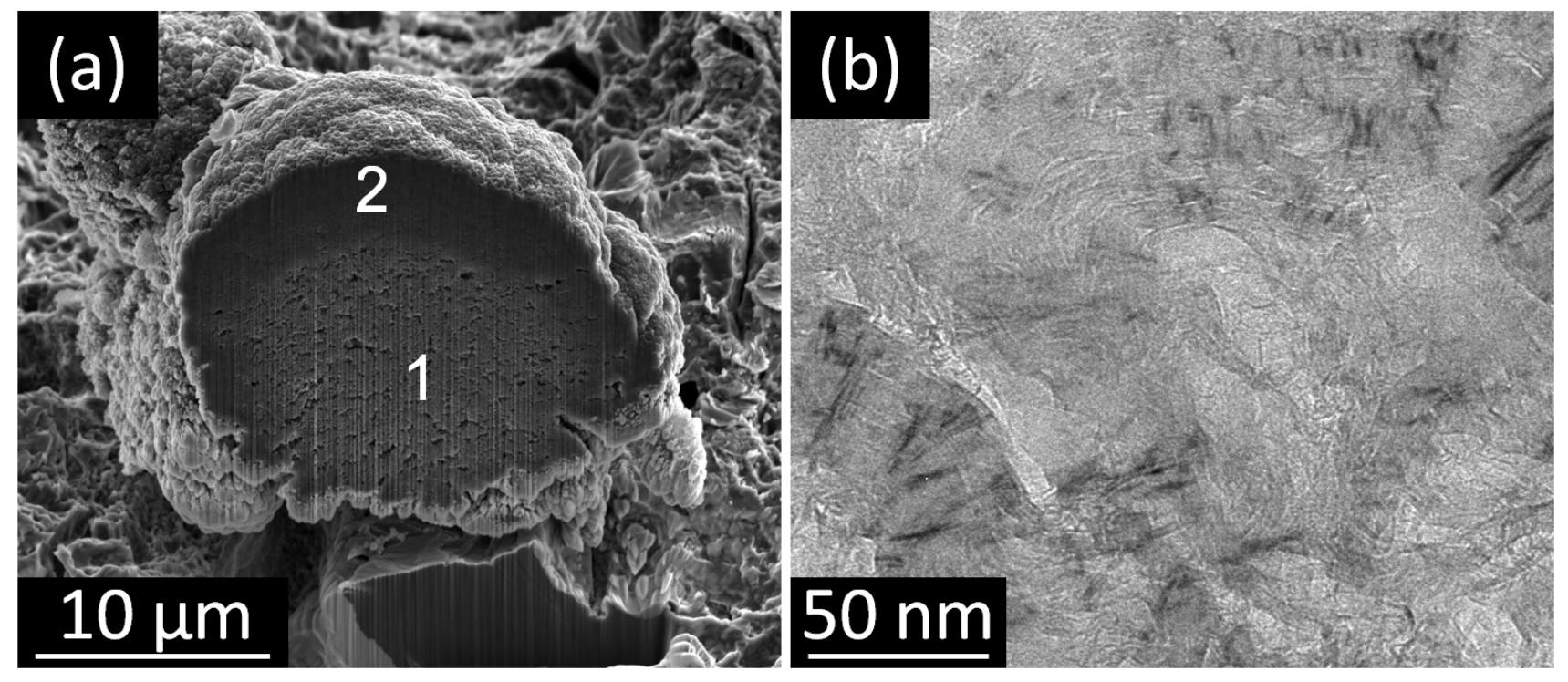




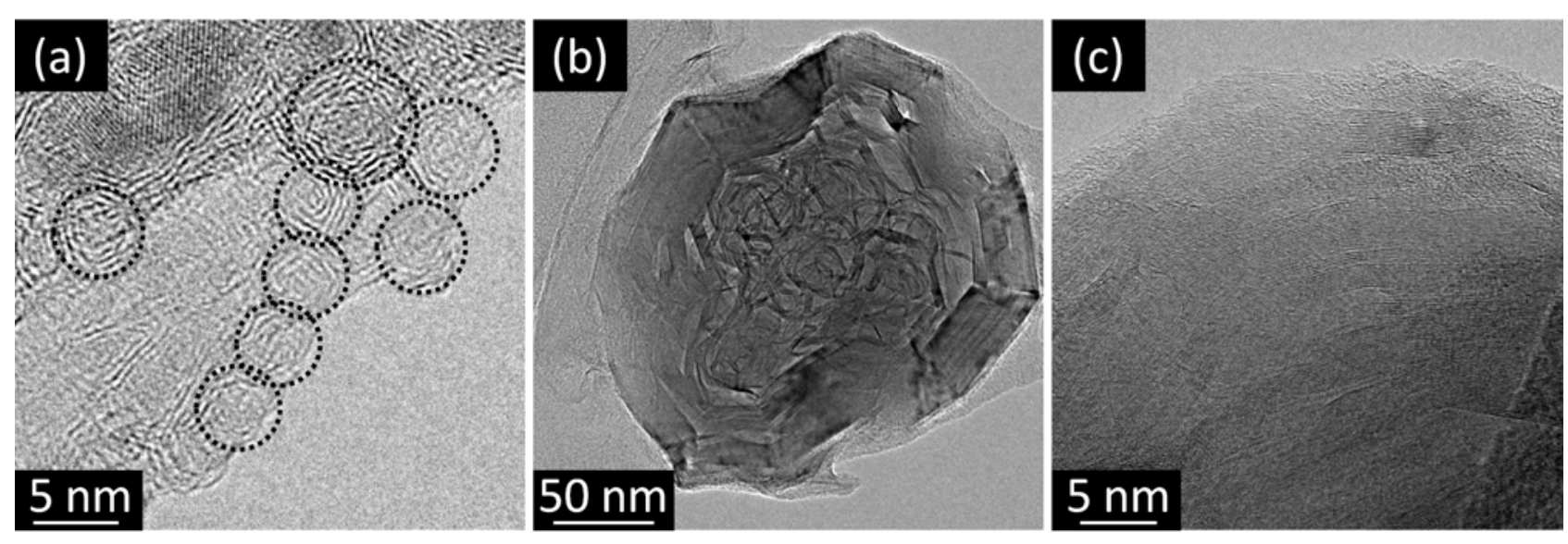



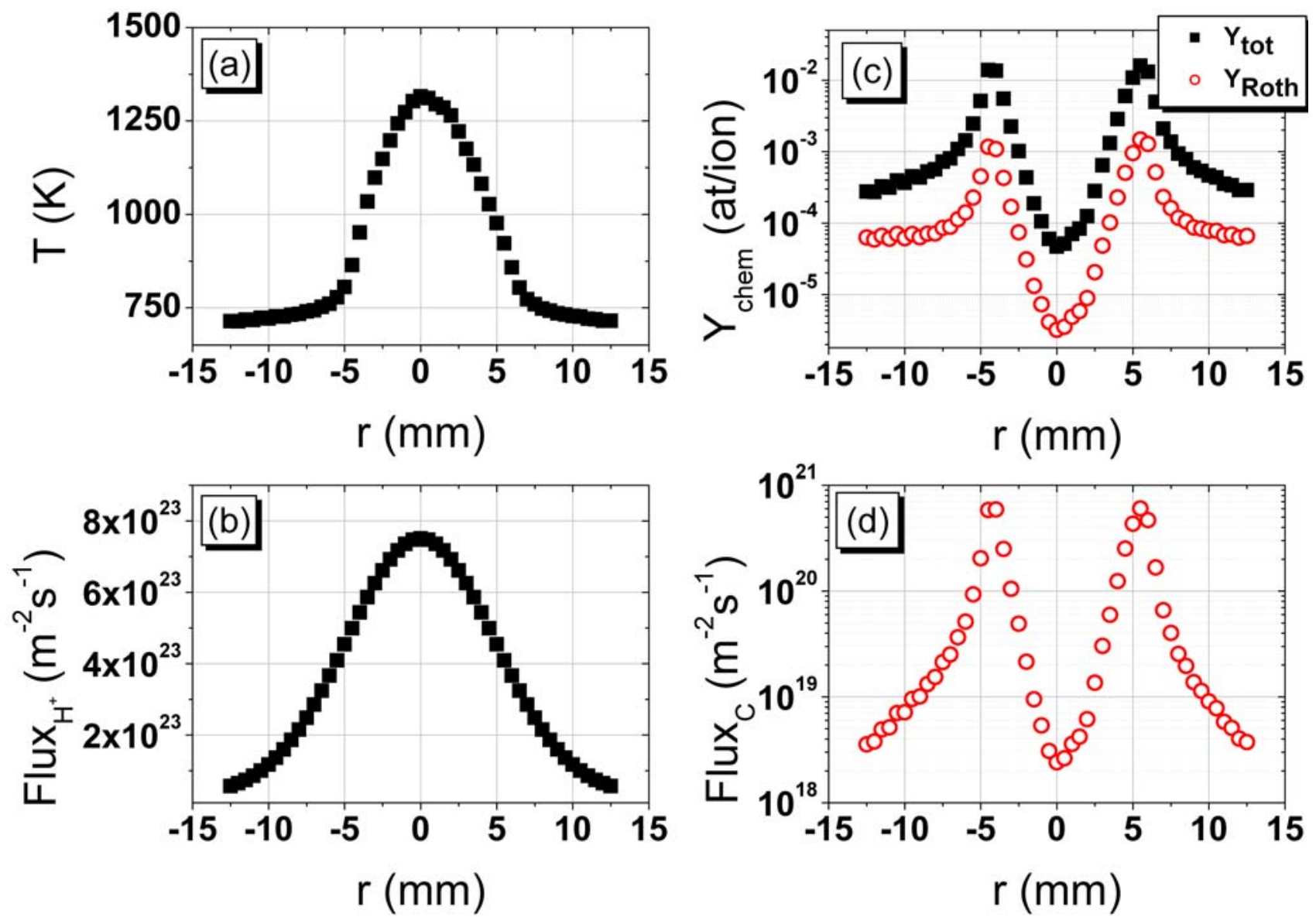